
2015

\title{
Biomolecules Editorial Office,
}

Published: 28 January 2016

MDPI AG, Klybeckstrasse 64, CH-4057 Basel, Switzerland; biomolecules@mdpi.com

The editors of Biomolecules would like to express their sincere gratitude to the following reviewers for assessing manuscripts in 2015.

We greatly appreciate the contribution of expert reviewers, which is crucial to the journal's editorial decision-making process. Several steps have been taken in 2015 to thank and acknowledge reviewers. Good, timely reviews are rewarded with a discount off their next MDPI publication. By creating an account on the submission system, reviewers can access details of their past reviews, see the comments of other reviewers, and download a letter of acknowledgement for their records. In addition, MDPI has launched a collaboration with Publons, a website that seeks to publicly acknowledge reviewers on a per journal basis. This is all done, of course, within the constraints of reviewer confidentiality. Feedback from reviewers shows that most see their task as a voluntary and mostly unseen work in service to the scientific community. We are grateful to our reviewers for the contribution they make.

Abbott, Karen L.

Achsel, Tilmann

Acuña, Darío

Agadjanyan, Michael

Amendola, Roberto

Amslinger, Sabine

Anifandis, Georgios M

Arbault, Stephane

Ariga, Hiroyoshi

Arteel, Gavin E

Artsimovitch, Irina

Askarian-Amiri, Marjan E.

Azuma, Miyuki

Babitzke, Paul

Baker, Mark D.

Balagurunathan, Kuberan

Banzola, Irina

Barabino, Silvia M. L.

Barbolina, Maria V.

Barrera, Giuseppina

Bartels, Tim
Hisanaga, Shin-ichi

Ho, Samuel

Hochrainer, Karin

Hoek, Jan B.

Hoffmann, Klaus

Holcik, Martin

Honke, Koichi

Hucke, Stephanie

Hughes, Michael

Iacono, Diego

Irwin, Jane

Ishigaki, Yasuhito

Ishino, Yoshizumi

James, Samet

Jamieson, Stephen M. F.

Janga, Sarath Chandra

Jasmin, Bernard

Jazirehi, Ali

JC, Rochet

Jeggo, Penny

Jens, Marvin
Quan, Taihao

Rademacher, Christoph

Rahman, Irfan

Ramakrishnan, Sadeesh

Ravid, Tommer

Reeves, Matthew

Ricciardelli, Carmela

Riess, Olaf

Rio, Donald

Riordan, James

Rizzarelli, Enrico

Robert, François

Rödel, Franz

Rodolfo, Carlo

Rodrigues, Pedro

Roman, Jesse

Roux, Philippe

Roychowdhury, Sanjoy

Rubin, Emanuel

Ryan, Barry

Rybalka, Emma 
Barzilay, Joshua I.

Basu, Amit

Bate, Clive

Baumann, Ulrich

Bayer, Peter

Bebb, Gwyn

Beisswenger, Paul J.

Bentley, David L.

Berman, Andrea

Beutler, Bruce

Bewick, Guy

Bleackley, Mark R.

Boag, Peter R.

Börner, Thomas

Bouaïcha, Noureddine

Brenner, J. Chad

Breydo, Leonid

Brosh, Robert M.

Brown, Susan

Bruno, Maurizio

Burgess, Richard R.

Burton, Zachary F.

Calado, Ângelo

Campbell, Christopher

Cannon, Martin

Carrier, France

Carter, Wayne G.

Carvalho, Beatriz

Castillo, Jose'

Caudle, Michael

Chandad, Fatiha

Chang, Hsueh-Wei

Chen, Jason J.

Chikindas, Michael L.

Chiocchetti, Roberto

Cho, Jae-Hyun

Choi, Kyung-Chul

Chowdhury, Indrajit

Ciccotosto, Joe

Cindrova-Davies, Tereza

Cisowsky, Jaroslaw

Clemens, Dahn
Jensen, Poul H.

Jensen, Roy A.

Jesus Raposo, Maria Filomena

Johansson, Åsa CM

Ju, Tongzhong

Kajiya, Hiroshi

Kalia, Lorraine

Kan, Lixin

Kanaar, Roland

Kanemori, Masaaki

Kanie, Osamu

Kapanidis, Achillefs

Kaplan, Craig

Karkhanis, Vrajesh

Kawaratani, Hideto

Keene, Jack

Khaliulin, Igor

Kharbanda, Kusum

Kiely, Aoife P.

Kim, Chang Guhn

Kimura, Yoshinobu

Kirpich, Irina A.

Kirsebom, Leif

Kitteringham, Neil

Klimas, Jan

Klis, Frans

Koeppen, Arnulf $\mathrm{H}$.

Kojima, Akiko

Kojo, Shosuke

Konopka, James B.

Kota, Satya

Kozlov, Andrey V.

Kracht, Michael

Krauss, Isaac J.

Kristensen, Peter

Kurokawa, Riki

Lacasse, Eric

Lawson, Mark

Lebaron, Richard

Lechel, Andre

Lee, Nam Ki

Lee, Tzong-shyuan
Saito, Mariko

Salmon, Adam

Samuel, Charles E.

Sancho-Bru, Pau

Sanij, Elaine

Saturnino, Carmela

Savage, Kienan I.

Schibli, Roger

Schleif, Robert

Schmid, Johannes A.

Schrum, Laura W.

Schubert, Mario

Schüller, Christoph

Schulz-Schaeffer, Walter

Schwartz, Christian

Seeling, Joni M.

Semsey, Szabolcs

Senchina, David S.

Serino, Giovanna

Seth, Devanshi

Sharma, Chander Shekhar

Sharma, Sudha

Sherwood, Roy A.

Shiloh, Yosef

Shimizu, Shigeomi

Shimizu, Toshiyuki

Sies, Helmut

Simmons, Rebecca A.

Simone, Giuseppina

Sinowatz, Fred

Slotboom, Dirk

Smart, Laura

Smiley, R. Derike

Smith, Christopher

Snapp, Erik Lee

Sobol, Robert W.

Son, Deok-soo

Souza-Smith, Flavia M.

Spencer, Juliet V.

Spoerri, Loredana

Spriano, Silvia

Staege, Martin S. 
Clugston, Robin

Costa, Max

Costantini, Maria

Cuppoletti, John

Curtin, Nicola J.

Curtis, Brenda

Daiber, Andreas

Darst, Seth A.

Davis, Terence

de Paz, Jose Luis

de Vries, Carlie

deHaseth, Pieter L.

Dehay, Benjamin

DeKrey, Gregory K.

Delle Site, Luigi

Di Fagagna, Fabrizio D'adda

Dikalov, Sergey

Ding, Wen-Xing

Domingues, Pedro

Donohue, Terrence

Duan, Zhenfeng

Duffield, Giles

Dulla, Chris G.

Duque, Paula

Ebright, Richard H.

Eckermann, Katrin

Eid, Nabil

Elmadhun, Nassrene

Everett, Gabby

Fähling, Michael

Falcone, Colomba

FANG, Evandro Fei

Fauvet, Bruno

La Favor, Justin D.

Feás, Xesús

Feklistov, Andrey

Feng, Wenke

Ferbeyre, Gerardo

Fernandes, Jason

Fernández-Solà, Joaquim

Ferrer, Isidro

Finazzi, Dario
Lehmann, Michael H.

Leich, Ellen

Lemieux, Hélène

Leng, Fenfei

Lewis, Dale E. A.

Li, Qingshun Quinn

Ligon, Lee A.

Liu, Jinsong

Liu, Chao

Ljungman, Mats

Loessner, Daniela

López, Claudia S.

Luco, Reini F

Ludovico, Paula

MacRae, Lola

Magalhaes, Ana

Maher, Louis J.

Malinski, Tadeusz

Mani, Ram

Maquat, Lynne E.

Martini, Maurizio

Mascher, Thorsten

Matias, Carlos M.

Mazin, Alexander

McCaskill, Michael

McFarland, Nikolaus R.

Mecerreyes, David

Medina, Miguel

Medzihradszky, Katalin F.

Mehta, Ashish

Mekler, Vladimir

Mercuri, Nicola Biagio

Meyer, Thomas F.

Michela, Castagna

Miller, Kyle M.

Mirey, Gladys

Moghe, Akshata

Moremen, Kelley W.

Morgan, Iain

Mothet, Jean-Pierre

Motohara, Takeshi

Mouradian, M. Maral
Stamm, Stefan

Stanley, Pamela

Starkov, Anatoly A.

Stec, David E.

Stocco, Carlos

Subramanian, Manikandan

Sue, Shih-Che

Sun, Shisheng

Sun, Chang Q.

Sundar, Isaac Kirubakaran

Sutherland, Greg T.

Suzuki, Tadashi

Svinartchouk, Fedor

Takeuchi, Tomoharu

Tan, Bertrand Chin-Ming

Tang, Hua

Tang, Petrus

Tansey, Malú G.

Tarantino, Giovanni

Tavazzi, Barbara

Taylor-Papadimitriou, Joyce

Teichmann, Sarah

Thapar, Roopa

Thatcher, Louise F.

Thorin-Trescases, Nathalie

Thornally, Paul J.

Tolmasky, Marcelo E.

Tomassetti, Antonella

Tomofuji, Takaaki

Trebicka, Jonel K.

Tucker, Steven

Tuma, Pamela

Umansky, Samuil

Vadigepalli, Rajanikanth

van der Velden, Adrianus W.M.

Van Sinderen, Douwe

van Waardenburg, Robert

Vancurova, Ivana

Vasiliou, Vasilis

Velasco Ortega, Eugenio

Verdin, Anthony

Verjee, Zulfikarali 
Fonseca, Bruno D.

Foray, Nicolas

Forni, Monica

Forsyth, Christopher B.

Franco, Rodrigo

Freeze, Hudson

Fritz, Kristofer S

Gadella, Bart M.

Gaillard, Hélène

Gao, Bin

García-Pavía, Pablo

Gelmann, Edward

Georgakilas, Alexandros G.

Gerber, André P.

Goldstein, Andrew S.

Gopalan, Venkat

Grallert, Beata

Greenwood, Michael T.

Guiza, Viviana

Haberl, Roman

Habib, Fouad K

Hams, Emily

Han, Dong Wook

Hanes, Steve

Hanson, Andrew

Haorah, James

Hartl, Dominik

Hartley, Rebecca

Hartmann, Marcus

Hatakeyama, Tomomitsu

Hautbergue, Guillaume

Hellstrom, Ingegerd

Helmann, John

Henao, Fernando

Herrmann, Johannes M.

Heyd, Florian

Hill, Michelle

Hirotsune, Shinji
Muñoz-Torrero, Diego

Munro, James

Murai, Junko

Murakami, Katsuhiko S.

Nagata, Eiichiro

Naito, Yuji

Nakada, Hiroshi

Natarajan, Sathish Kumar

O'Connell, Matthew

Odum, Niels

Ogawa, Tomohisa

Ogino, Shuji

Ohno, Kinji

Öhrfelt, Annika

Oka, Shogo

Okamura, Nobuyuki

Osaka, Tetsuya

Otzen, D.E.

Pabst, Martin

Padler-Karavani, Vered

Paller, Channing

Paré, Paul W.

Pattathil, Sivakumar

Pei, Jin-Jing

Pelletier, Guillaume

Peng, Wen-Huang

Pereira, C.

Pesti, Miklós

Pfander, Boris

Pócsi, István

Poisson, Thomas

Polanco, Juan Carlos

Poluektova, Larisa

Pountney, Dean

Prentice, Howard

Price, Clive

Pritchard, Michelle

Prosperi, Ennio
Vermeulen, Wim

Vila, Miquel

Vogel, Christoph

Voncken, Jan Willem

Voutquenne, Laurence

Wang, Peng

Wang, Isabel

Warriner, Stuart L.

Watanabe, Yuichiro

Watkins, Stephanie

Wiemer, Erik A. C.

Wilma, Olson

Wilson, Melanie

Witt, Steffan

$\mathrm{Wu}$, Shih-Hsiung

Wuhrer, Manfred

Wyatt, Todd

Xing, Daisy D.

$\mathrm{Xu}$, Peng

$\mathrm{Xu}$, Yongjie

Yamada, Shoji

Yamagata, Masaki

Yan, Shan

Yang, Yongguang

Yao, Yanhua

Yeligar, Samantha

Yin, Lei

Yin, Xiao-ming

Yokoyama, Shigeyuki

$\mathrm{Yu}$, Bin

Zahnow, Cynthia Ann

Zandberg, Wesley

Zhang, Fuming

Zhou, Zhanxiang

Zoidis, Evangelos

Zulli, A.

Zwart, Sara

Zwollo, Patty

(C) 2016 by the author; licensee MDPI, Basel, Switzerland. This article is an open access article distributed under the terms and conditions of the Creative Commons by Attribution (CC-BY) license (http://creativecommons.org/licenses/by/4.0/). 